University of Wollongong

Research Online

Faculty of Engineering and Information

Faculty of Engineering and Information

Sciences - Papers: Part A

Sciences

$1-1-2013$

On-chip high sensitivity laser frequency sensing with Brillouin mutuallymodulated cross-gain modulation

\author{
Feng Gao \\ University of Sydney, fgao@uow.edu.au \\ R Pant \\ University Of Sydney \\ Enbang Li \\ University of Wollongong, enbang@uow.edu.au \\ Christopher G. Poulton \\ University of Technology, Sydney \\ D Choi \\ Australian National University
}

See next page for additional authors

Follow this and additional works at: https://ro.uow.edu.au/eispapers

Part of the Engineering Commons, and the Science and Technology Studies Commons

Research Online is the open access institutional repository for the University of Wollongong. For further information contact the UOW Library: research-pubs@uow.edu.au 


\title{
On-chip high sensitivity laser frequency sensing with Brillouin mutually- modulated cross-gain modulation
}

\author{
Abstract \\ We report the first demonstration of a photonic-chip laser frequency sensor using Brillouin mutually- \\ modulated cross-gain modulation (MMXGM). A large sensitivity $(\sim 9.5 \mathrm{mrad} / \mathrm{kHz})$ of the modulation \\ phase shift to probe carrier frequency is demonstrated at a modulation frequency of $50 \mathrm{kHz}$ using \\ Brillouin MMXGM in a $\sim 7 \mathrm{~cm}$ long chalcogenide rib waveguide. (c) 2013 Optical Society of America.

\section{Keywords} \\ cross, modulation, modulated, gain, mutually, brillouin, sensing, frequency, laser, sensitivity, high, chip \\ Disciplines \\ Engineering | Science and Technology Studies

\section{Publication Details} \\ Gao, F., Pant, R., Li, E., Poulton, C. G., Choi, D., Madden, S. J., Luther-Davies, B. \& Eggleton, B. (2013). On- \\ chip high sensitivity laser frequency sensing with Brillouin mutually-modulated cross-gain modulation. \\ Optics Express, 21 (7), 8605-8613.

\section{Authors} \\ Feng Gao, R Pant, Enbang Li, Christopher G. Poulton, D Choi, Stephen J. Madden, B Luther-Davies, and \\ Benjamin Eggleton
}




\title{
On-chip high sensitivity laser frequency sensing with Brillouin mutually-modulated cross-gain modulation
}

\author{
Feng Gao, ${ }^{1,2, *}$ Ravi Pant, ${ }^{1}$ Enbang Li, ${ }^{1,3}$ Christopher G. Poulton, ${ }^{4}$ \\ Duk-Yong Choi,${ }^{5}$ Stephen J. Madden, ${ }^{5}$ Barry Luther-Davies, ${ }^{5}$ and \\ Benjamin J. Eggleton ${ }^{1}$ \\ ${ }^{1}$ Centre for Ultrahigh bandwidth Devices for Optical Systems (CUDOS), Institute of \\ Photonics and Optical Science (IPOS), School of Physics, The University of Sydney, New \\ South Wales 2006, Australia \\ ${ }^{2}$ MOE Key Laboratory of Weak-Light Nonlinear Photonics, TEDA Applied Physics School and \\ School of Physics, \\ Nankai University, Tianjin 300457, China \\ ${ }^{3}$ School of Engineering Physics, University of Wollongong, New South Wales 2522, Australia \\ ${ }^{4}$ School of Mathematical Sciences, University of Technology Sydney, NSW 2007, Australia \\ ${ }^{5}$ CUDOS, Laser Physics Centre, Australian National University, Canberra, Australian Capital \\ Territory 0200, Australia \\ *fenggao@nankai.edu.cn
}

\begin{abstract}
We report the first demonstration of a photonic-chip laser frequency sensor using Brillouin mutually-modulated cross-gain modulation (MMXGM). A large sensitivity $(\sim 9.5 \mathrm{mrad} / \mathrm{kHz})$ of the modulation phase shift to probe carrier frequency is demonstrated at a modulation frequency of $50 \mathrm{kHz}$ using Brillouin MMXGM in a $\sim 7 \mathrm{~cm}$ long chalcogenide rib waveguide.
\end{abstract}

(C) 2013 Optical Society of America

OCIS codes: (120.5050) Phase measurement; (190.0190) Nonlinear optics; (190.2640) Stimulated scattering, modulation, etc.; (190.4360) Nonlinear optics, devices.

\section{References and links}

1. O. P. Lay, S. Dubovitsky, R. D. Peters, J. P. Burger, S. W. Ahn, W. H. Steier, H. R. Fetterman, and Y. Chang, "MSTAR: a submicrometer absolute metrology system," Opt. Lett. 28, 890-892 (2003).

2. Z. Xie and H. F. Taylor, "Fabry-Perot optical binary switch for aircraft applications," Opt. Lett. 31, 2695-2697 (2006).

3. X. F. Mo, B. Zhu, Z. F. Han, Y. Z. Gui, and G. C. Guo, "Faraday-Michelson system for quantum cryptography," Opt. Lett. 30, 2632-2634 (2005).

4. S. Sakadzic and L. V. Wang, "High-resolution ultrasound-modulated optical tomography in biological tissues," Opt. Lett. 29, 2770-2772 (2004).

5. Z. Shi, R. W. Boyd, D. J. Gauthier, and C. C. Dudley, "Enhancing the spectral sensitivity of interferometers using slow-light media," Opt. Lett. 32, 915-917 (2007).

6. Z. Shi and R. W. Boyd, "Slow-light interferometry: practical limitations to spectroscopic performance," J. Opt. Soc. Am. B, 25, C136-C143 (2008).

7. L. Thévenaz, "Slow and fast light in optical fibres," Nat. Photonics 2, 474-481 (2008).

8. M. González-Herráez, K. Song, and L. Thévenaz, "Optically controlled slow and fast light in optical fibers using stimulated Brillouin scattering,” AppL. Phys. Lett. 87, 081113 (2005).

9. Y. Okawachi, M. S. Bigelow, J. E. Sharping, Z. Zhu, A. Schweinsberg, D. J. Gauthier, R. W. Boyd, and A. L. Gaeta, "Tunable All-Optical Delays via Brillouin Slow Light in an Optical Fiber," Phys. Rev. Lett. 94, 153902 (2005). 
10. R. Pant, M. D. Stenner, M. A. Neifeld, and D. J. Gauthier, "Optimal pump profile designs for broadband SBS slow-light systems," Opt. Express 16, 2764-2777 (2008).

11. R. Pant, A. Byrnes, C. G. Poulton, E. Li, D. Choi, S. Madden, B. Luther-Davies, and B. J. Eggleton, "Photonicchip-based tunable slow and fast light via stimulated Brillouin scattering," Opt. Lett. 37, 969-971 (2012).

12. T. Arditi, E. Granot, and S. Sternklar, "Nonlinear phase shifts of modulated light waves with slow and superluminal group delay in stimulated Brillouin scatting," J. Opt. 12, 104016 (2010).

13. S. Sternklar, E. Sarid, A. Arbel, and E. Granot, "Brillouin cross-gain modulation and $10 \mathrm{~m} / \mathrm{s}$ group velocity," Opt. Lett. 34, 2832-2834 (2009).

14. S. Sternklar, E. Sarid, M. Wart, and E. Granot, "Mutually-modulated cross-gain modulation and slow light," J. Opt. 12, $104016(2010)$

15. S. Sternklar, M. Vart, A. Lifshitz, S. Bloch, and E. Granot, "Kilohertz laser frequency sensing with Brillouin mutually modulated cross-gain modulation," Opt. Lett. 36, 4161-4163 (2011)

16. R. Pant, C. G. Poulton, D.Choi, H. Mcfarlane, S. Hile, E. Li, L. Thevenaz, B. Luther-Davies, S. J. Madden, and B. J. Eggleton, “On-chip stimulated Brillouin scattering,” Opt. Express 19, 8285-8290 (2011).

17. B. J. Eggleton, T. D. Vo, R. Pant, J. Schroeder, M. D. Pelusi, D. Yong Choi, S. J. Madden, and B. Luther-Davies, "Photonic chip based ultrafast optical processing based on high nonlinearity dispersion engineered chalcogenide waveguides," Laser Photonics Rev., 6, Issue 1, 97-114 (2012).

18. B. J. Eggleton, B. Luther-Davies, and K. Richardson, "Chalcogenide photonics," Nat. Photonics, 5, 141-148 (2011).

19. K. Qian, L. Zhan, L. Zhang, Z. Q. Zhu, J. S. Peng, Z. C. Gu, X. Hu, S. Y. Luo, and Y. X. Xia, “Group velocity manipulation in active fibers using mutually modulated cross-gain modulation: from ultraslow to superluminal propagation," Opt. Lett. 36, 2185-2188 (2011).

\section{Introduction}

Devices with large spectral sensitivity are becoming increasingly desirable in applications such as metrology, optical sensing, quantum information processing, and biomedical engineering [1-4]. Although the spectral sensitivity can be enhanced using slow light $[5,6]$, the central measurement is usually that of the optical phase, and therefore requires the use of an interferometer. Recently, Sternklar et al. exploited a novel slow-light configuration known as Brillouin mutually-modulated cross-gain modulation (MMXGM). In this configuration, both a pump and Stokes signal are modulated at the same frequency; the Brillouin gain then introduces higher harmonics to the Stokes signal, with the first harmonic possessing a phase delay that is extremely sensitive to the value of the gain. By measuring the phase delay of the first harmonic, an extremely sensitive measurement of the detuning of the Stokes shift may be obtained. This technique relies on the gain associated with the Brillouin resonance rather than the dispersion, and so differs from frequency measurements that directly exploit the presence of slow- and fast-light near an SBS resonance [7-14]. With such a configuration, an impressive spectral sensitivity $\sim 6.5 \mathrm{mrad} / \mathrm{kHz}$ in the modulation phase was achieved for a modulation frequency of $100 \mathrm{~Hz}$ in a $3 \mathrm{~km}$ long optical fiber [15]. The striking feature of this configuration is that it does not require an interferometer: the spectral sensitivity is enhanced in the modulation phase rather than the optical phase, which makes it convenient for many optical applications.

Recently, on-chip stimulated Brillouin scattering was demonstrated using the large Brillouin scattering cross-section $\left(g_{B}=0.74 \times 10^{-9} \mathrm{~m} / \mathrm{W}\right)$, small effective mode area $\left(A_{\text {eff }}=2.3 \mu \mathrm{m}^{2}\right)$ and strong light-sound confinement in a $\sim 7 \mathrm{~cm}$ chalcogenide $\left(\mathrm{As}_{2} \mathrm{~S}_{3}\right)$ rib waveguide [16]. Here, we report the first demonstration of photonic-chip laser frequency sensing using Brillouin MMXGM. For our on-chip Brillouin MMXGM demonstration a large spectral sensitivity of $\sim 9.5 \mathrm{mrad} / \mathrm{kHz}$ was measured for a modulation frequency of $50 \mathrm{kHz}$ - this absolute sensitivity is comparable to the previous MMXGM-based measurements in kilometers of fiber. It has been noted in previous work, that provided the phase shift between the pump and Stokes signal can be made exactly equal to $\pi$ radians, the sensitivity is inversely proportional to the length of the gain medium [15]. This suggests that on-chip devices that use Brillouin MMXGM can potentially be used to significantly enhance the achievable sensitivity. On-chip implementation of Brillouin MMXGM is also desirable from the point of view of the creation of compact on-chip 
integrated photonics devices. We discuss here the dependence of the sensitivity on the device length as well as on deviation from the phase shift of $\pi$, and show that the maximum spectral sensitivity is also limited by the resolution achievable in controlling the phase of the modulated Stokes signal.

\section{Principle and theory}

Figure 1 shows the concept of laser frequency sensing using Brillouin MMXGM in a chalcogenide $\left(\mathrm{As}_{2} \mathrm{~S}_{3}\right)$ photonic-chip $[17,18]$. A pump $\left(\omega_{p}\right)$ and a Stokes signal $\left(\omega_{s}\right)$ are both modulated at the same frequency $f_{\text {mod }} \ll f_{\text {Brillouin }}$ and are counter-propagated through the chip. If the frequency difference between pump and Stokes signals is close to the Brillouin resonant frequency $f_{\text {Brillouin }}$ then the Stokes will experience Brillouin gain, and will undergo selective amplification at the points where the peaks of the modulated pump coincide with the troughs of the modulated Stokes. This effect distorts the Stokes wave and introduces higher harmonics. The first harmonic wave possesses a phase delay which is extremely sensitive to the detuning from the Brillouin resonance. It is important to note that the phase modulation due to MMXGM is fundamentally different from the phase changes induced by the dispersion of SBS, and can be made extremely sensitive to frequency detuning.

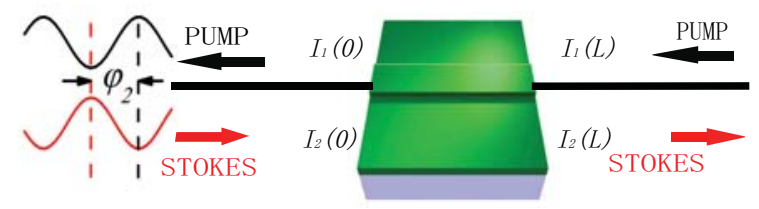

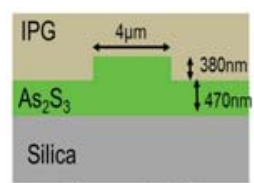

Photonic chip

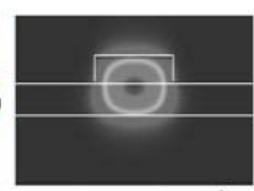

Acoustic mode

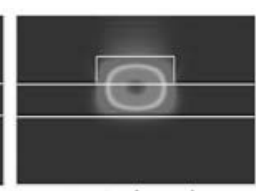

Optical mode

Fig. 1. MMXGM in the SBS medium. Both pump and signal are intensity modulated and the modulation phase shift at the front (left) facet is $\varphi_{2}$.

The theory and principle of laser frequency sensing using Brillouin MMXGM has been previously derived in [14], for the specific case in which the modulation phase difference $\varphi_{2}$ between pump and Stokes is equal to $\pi$. In the following we give an outline of this theory, both to clarify the underlying physics and also to discuss the effects of deviations of $\varphi_{2}$ from the exact value of $\pi$.

Assuming a modulation depth of $\alpha$ for the pump signal, the pump intensity $I_{1}(z, t)$ can be written at the end of the waveguide $(z=L)$ as:

$$
I_{1}(L, t)=I_{1}^{0}[1+\alpha \cos (K L+\Omega t)],
$$

where $\Omega=2 \pi f_{\text {mod }}$ is the angular modulation frequency and $K=\Omega n / c$ is the modulation wavenumber for a waveguide with effective index $n$. The Stokes signal is modulated with modulation depth $\beta$. At the front facet $(z=0)$ the intensity $I_{2}(z, t)$ of the modulated Stokes is:

$$
I_{2}(0, t)=I_{2}^{0}\left[1+\beta \cos \left(-\varphi_{2}-\Omega t\right)\right],
$$

where $\varphi_{2}$ is the modulation phase shift between the pump and the Stokes signals at the front facet of the sample as shown in Fig. 1. As it propagates through the waveguide the Stokes signal 
experiences Brillouin gain. Under the approximation that the pump is undepleted, at the output facet $(z=L)$ the Stokes signal can be written [14]:

$$
I_{2}(z=L, t)=I_{2}^{0}\left[1+\beta \cos \left(K L-\Omega t-\varphi_{2}\right)\right] \exp [G+\alpha G \operatorname{sinc}(K L) \cos (\Omega t)],
$$

where the gain $G$ depends on detuning $\delta \omega_{s}$ from the Brillouin maximum according to the Lorentzian function:

$$
G=G_{0}\left[1+\left(\frac{2 \delta \omega_{s}}{\Gamma_{B}}\right)^{2}\right]^{-1}
$$

where $G_{0}=g_{B} I_{1}^{0} L$ is the peak gain and $\Gamma_{B}$ is the Brillouin linewidth, which is typically on the order of $10-50 \mathrm{MHz}$ in most optical materials.

The expression for the amplified Stokes can be expressed equivalently as a sum of harmonics:

$$
I_{2}(L, t)=i_{D C}+i_{1} \cos \left(\theta_{1}-\Omega t\right)+i_{2} \cos \left(\theta_{2}-2 \Omega t\right)+\cdots
$$

where the coefficients $i_{m}(m \geq 1)$ and phases $\theta_{m}$ are functions of $K, L, \alpha, \beta, G$ and $\varphi_{2}$. With weak modulation and $K L \ll 1$, Eq. (3) can be expanded as

$$
I_{2}(L, t) \simeq I_{2}^{0} \exp (G)\left[1+\beta \cos \left(K L-\Omega t-\varphi_{2}\right)+\alpha G \operatorname{sinc}(K L) \cos (\Omega t)\right] .
$$

In Eq. (6), the terms on the right are the DC gain of the Stokes, the modulation from the Stokes and the modulation from the pump, respectively.

The total phase shift of the first harmonic $\theta_{1}$ is

$$
\theta_{1}=K L+\tan ^{-1}\left(\frac{\hat{G} K L+\sin \left(\varphi_{2}\right)}{-\cos \left(K L-\varphi_{2}\right)-\hat{G}}\right)
$$

where $\hat{G}=G \alpha / \beta$. In Eq. (7), the $K L$ term represents the linear phase delay across the propagation medium and the inverse tangent denotes the nonlinear phase delay that occurs as a result of Brillouin MMXGM. This effect depends on the detuning of the central Stokes peak from the center of the Brillouin resonance, as given by Eq. (4). This change in the gain will in turn advance or delay the phase of the first harmonic. The spectral sensitivity $S$ depends directly on the change in the first-harmonic phase $\Delta \theta_{1}$ when there is a change in $\delta \omega_{s}$ :

$$
S:=\frac{d \theta_{1}}{d\left(\delta \omega_{s}\right)}=\frac{d \theta_{1}}{d \hat{G}} \frac{d \hat{G}}{d\left(\delta \omega_{s}\right)}=\frac{-K L \cos \varphi_{2}+\sin \varphi_{2}}{\left(\cos \varphi_{2}+\hat{G}\right)^{2}+\left(\hat{G} K L+\sin \varphi_{2}\right)^{2}} \frac{d \hat{G}}{d\left(\delta \omega_{s}\right)}
$$

From Eq. (8) it can be seen that when $\varphi_{2}=\pi$, the maximum sensitivity at $\hat{G} \approx 1$ will be inversely proportional to both $L$ and $f_{\text {mod }}$, yielding the same result as previously derived in [14] and [15]. In principle, provided an exact phase modulation shift of $\varphi_{2}=\pi$ can be reached, extremely high sensitivities can be attained by using short waveguides and low modulation frequencies. In practice, however, there is a limit to the resolution with which the modulation phase can be determined. We now consider the modulation phase shift to be close but not equal to $\pi$, such that $\varphi_{2}=\pi+\delta \varphi_{2}$, where $\delta \varphi_{2}$ is small. Continuing the assumption that $K L<<1$, we find from Eq. (8) that the maximum sensitivity is

$$
S_{\max } \approx \frac{1}{K L-\delta \varphi_{2}} \frac{d \hat{G}}{d\left(\delta \omega_{s}\right)}
$$

This result shows that the limitation on the maximum sensitivity is determined by the larger of the quantities $K L$ and $\delta \varphi_{2}$. We note that in Eq. (9), the sensitivity changes sign when $K L \sim \delta \varphi_{2}$. This corresponds to a transition between the slow light and fast light regions $[13,19]$. Because 
$K L$ is always positive, the situation when $\varphi_{2}$ is exactly equal to $\pi$ corresponds to the slow-light configuration.

As noted previously [15], there exist two sensing windows, spaced symmetrically around the Stokes frequency. Within each sensing window, and provided that $K L$ is small, the phase shift $\theta_{1}$ varies monotonically with $\delta \omega_{s}$ between either zero and $\pi$, for the slow-light configuration, or between zero and $-\pi$ for the fast-light configuration. At the position of maximum sensitivity, which occurs in the middle of the sensing window, $\theta_{1}$ is equal to $\pm \pi / 2$.

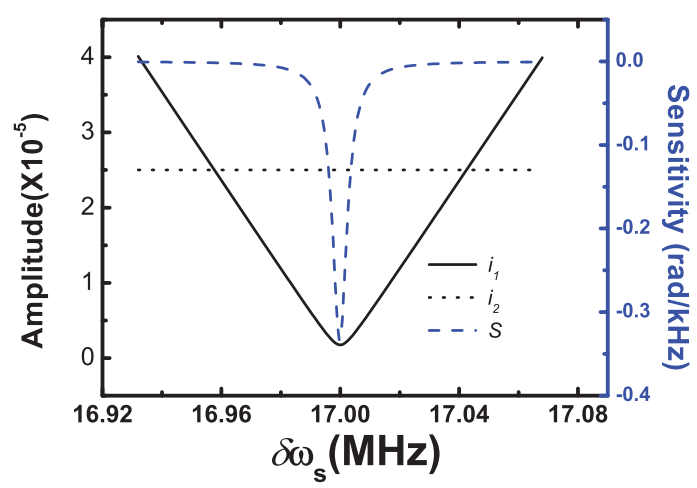

Fig. 2. Calculated amplitude of the first order harmonic wave $i_{1}$ (black solid line) and the amplitude of the second order harmonic wave $i_{2}$ (black dotted line) at different sensitivity with $\hat{G}_{0}=2$ and $\varphi_{2}=\pi$. The blue dash curve shows the sensitivity $S$.

From the above analysis, we note that Eq. (7) for the modulation phase shift was derived assuming only the first-order harmonic. However, the actual output Stokes wave (see Eq. (3) and Eq. (5)) contains higher-order harmonics. Figure 2 shows the theoretically calculated amplitudes of first- and second-order harmonics around the sensing window (centered around $17 \mathrm{MHz}$ ) as calculated using Eq. (10) and Eq. (14) from [14], for a $7 \mathrm{~cm}$ chalcogenide ribwaveguide used in the experiments. For these calculations we used the following parameters: $n=2.4, \Gamma_{B}=34 \mathrm{MHz}, f_{\text {mod }}=50 \mathrm{kHz}, \hat{G}_{0}=\alpha G_{0} / \beta=2$ and $\varphi_{2}=\pi$. From Fig. 2 , we note that the amplitude of the second-order harmonic is larger than that of the fundamental in the sensing window where the spectral sensitivity $S$ is large. Therefore, in order to measure the phase change of first harmonic wave, higher-order harmonics must be removed in the experiment. In our experiments, we used an electrical filter to remove the higher harmonics.

The calculations shown in Fig. 2 were performed for a sensing window centered around $17 \mathrm{MHz}$. However, the position of the sensing window, defined by the detuning of its center frequency from the peak of the Brillouin resonance, varies with the value of $\hat{G}_{0}$ [15]. In Fig. 3 we show the calculated position of the sensing window and the corresponding maximum sensitivity $S$ for different values of $\hat{G}_{0}$. Typically the sensing window is a narrow frequency region around the center frequency. However, it is possible to apply the sensing technique in a large frequency range around the the peak of the Brillouin resonance by changing the value of $\hat{G}_{0}$. From Fig. 3, we note that when $\hat{G}_{0}$ increased from 1.5 to 5.5 , the maximum sensitivity varies slowly, changing less than $25 \%$. 


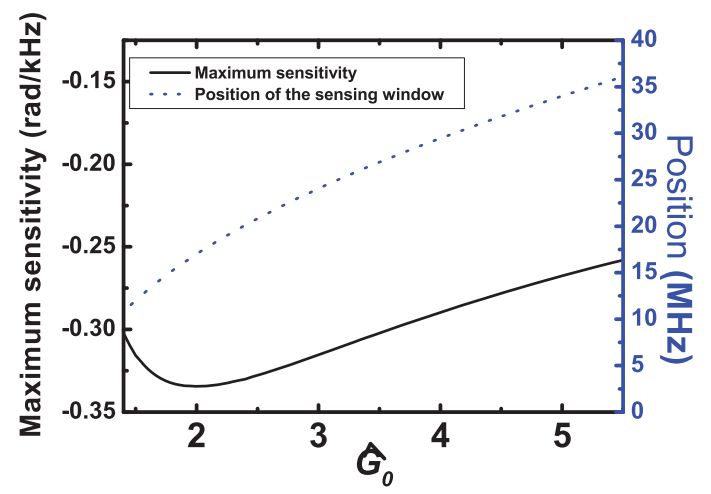

Fig. 3. Calculated maximum sensitivity (black solid line) and position of the sensing window (blue dotted line) with different $\hat{G}_{0}$.

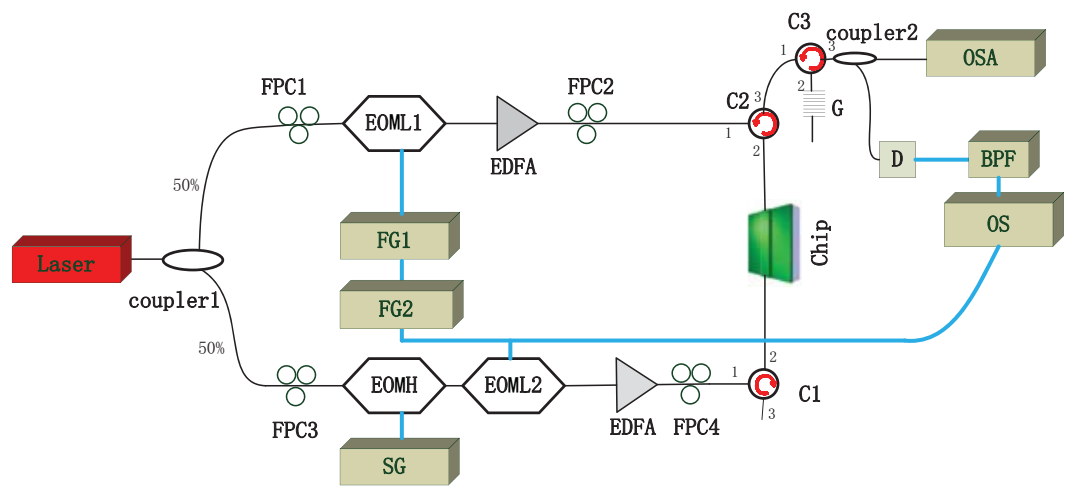

Fig. 4. The configuration of the experiment. FG1 and FG2 are function generators; FPC1-4 is the fiber polarization controller; $\mathrm{SG}$ is the signal generator to create Stokes wave; $\mathrm{C}$, $\mathrm{C} 2$ and $\mathrm{C} 3$ are circulators; EOML1, EOML2 and EOMH are intensity modulators; EDFA is the Erbrium doped fiber amplifier; OSA is the optical spectrum analyzer; D is optical detector; BPF is electronic band pass filter and OS is the oscilloscope. Blue wires indicate the electronic wire. 


\section{Experiment}

Figure 4 shows the experimental set-up for the on-chip Brillouin MMXGM sensor. A tunable laser around $1550 \mathrm{~nm}$ was split with a 50/50 coupler to generate the pump and Stokes wave. After the Stokes signal was generated in one arm using the intensity modulator EOMH (electrooptic modulator of high frequency) driven by a signal generator SG, the light in both the arms was sinusoidally modulated by intensity modulators EOML1 and EOML2 (electro-optic modulator of low frequency) driven by function generators FG1 and FG2 at $50 \mathrm{kHz}$. The modulation phase could be controlled by adjusting the phase delay from the function generator FG2 triggered by FG1. Both the pump and signal were amplified by erbium doped fiber amplifiers (EDFAs) and coupled to a $7 \mathrm{~cm}$ long waveguide with a cross section $4 \mu \mathrm{m} \times 850 \mathrm{~nm}$ through circulators $(\mathrm{C} 1, \mathrm{C} 2)$ using lensed fibers. The total insertion loss was around $13 \mathrm{~dB}$ during the experiment, measured through port 3 of $\mathrm{C} 1$. The amplified Stokes signal was sent into a circulator $\mathrm{C} 3$ with a Bragg grating connected to port 2 (see Fig. 4). The 3dB-bandwidth of the Bragg grating was about $0.13 \mathrm{~nm}$ and one of its steep edges of the reflection peak was used to suppress the parasitic pump originated from the reflection at the chip input facet. The filtered signal was split using a $99 / 1$ coupler where the $1 \%$ port was sent to the OSA and the $99 \%$ port was sent to a photo detector D. An electronic band pass filter BPF with a $2 \mathrm{kHz}$ bandwidth was used before the detector to filter out the higher harmonics and DC component. The electronically filtered signal was observed on the oscilloscope OS.

From Fig. 3, we note that the frequency at which the maximum sensitivity occurs depends on the value of $\hat{G}_{0}$, which enables the application of this technique over a large frequency range around the peak of the Brillouin resonance $(\sim 7.63 \mathrm{GHz})$. In our experiment, we first adjusted the pump and Stokes polarization to achieve a maximum gain $(\sim 5-6 \mathrm{~dB})$ at the peak of the Brillouin gain while keeping $\alpha \approx \beta \approx 0$. In order to show that we can achieve large sensitivity for any frequency around the peak of the Brillouin resonance by adjusting the value of $\hat{G}_{0}$, we arbitrarily picked a frequency detuning of $\Delta\left(\delta \omega_{s}\right) \sim 25 \mathrm{MHz}$ up-shifted from the peak of the Brillouin resonance. By observing the pump and Stokes waveform on OS, at port 2 of $\mathrm{C} 1$, we set $\varphi_{2}=\pi$. The value of $\hat{G}_{0}$ was then varied by adjusting the modulation depths $\alpha$ and $\beta$ (whilst keeping them small) so that $\theta_{1}=\pi / 2$, which corresponds to the maximum sensitivity, occurs at $\Delta\left(\delta \omega_{s}\right) \sim 25 \mathrm{MHz}$. Finally, we measured the sensitivity for a range of different values of $\varphi_{2}$ around a detuning $\delta \omega_{s} \sim 25 \mathrm{MHz}$, and determined the value of $\varphi_{2}$ for which the sensitivity was maximized. With this optimized value of $\varphi_{2}$, the frequency of the Stokes was detuned from $\delta \omega_{s} \sim 25 \mathrm{MHz}$ in steps of $100 \mathrm{kHz}$ to observe the phase shift evolution of the first-harmonic on OS. In our experiments, the maximum sensitivity was obtained for a phase evolution from $-\pi$ to 0 , therefore occurring in the fast-light regime. We also observed phase evolution from the slow-light regime of 0 to $\pi$, however the sensitivity at this value of $\varphi_{2}$ was smaller. In the following we present here the results for the overall maximum sensitivity from the fast-light regime.

Figure 5 shows the output waveform recorded on OS with the largest sensitivity we could obtain at the modulation frequency of $50 \mathrm{kHz}$, together with the normalized phase shift of the first harmonic wave. The black dashed curve in the center of Fig. 5(A) shows the evolution of the phase when the frequency detuning was changed. A phase shift evolution from $-\pi$ to 0 was observed when the frequency was detuned from $24.876 \mathrm{MHz}$ to $25.876 \mathrm{MHz}$ in steps of 100 $\mathrm{kHz}$. In our experiment, we observed a small distortion arising from the residual higher-order harmonics remaining after the band pass filter. The normalized phase shift plotted in Fig. 5 (B) shows a phase shift from $-\pi$ to 0 when the frequency was detuned away from the peak of the Brillouin resonance. A maximum phase shift of $\Delta \theta_{1} \sim 0.95 \mathrm{rad}$ was observed around detuning of $25.476 \mathrm{MHz}$ (see Fig. 5(B)), corresponding to a maximum sensitivity of $\sim 9.5 \mathrm{mrad} / \mathrm{kHz}$. The maximum attainable sensitivity is limited by the ability to measure the phase change of 

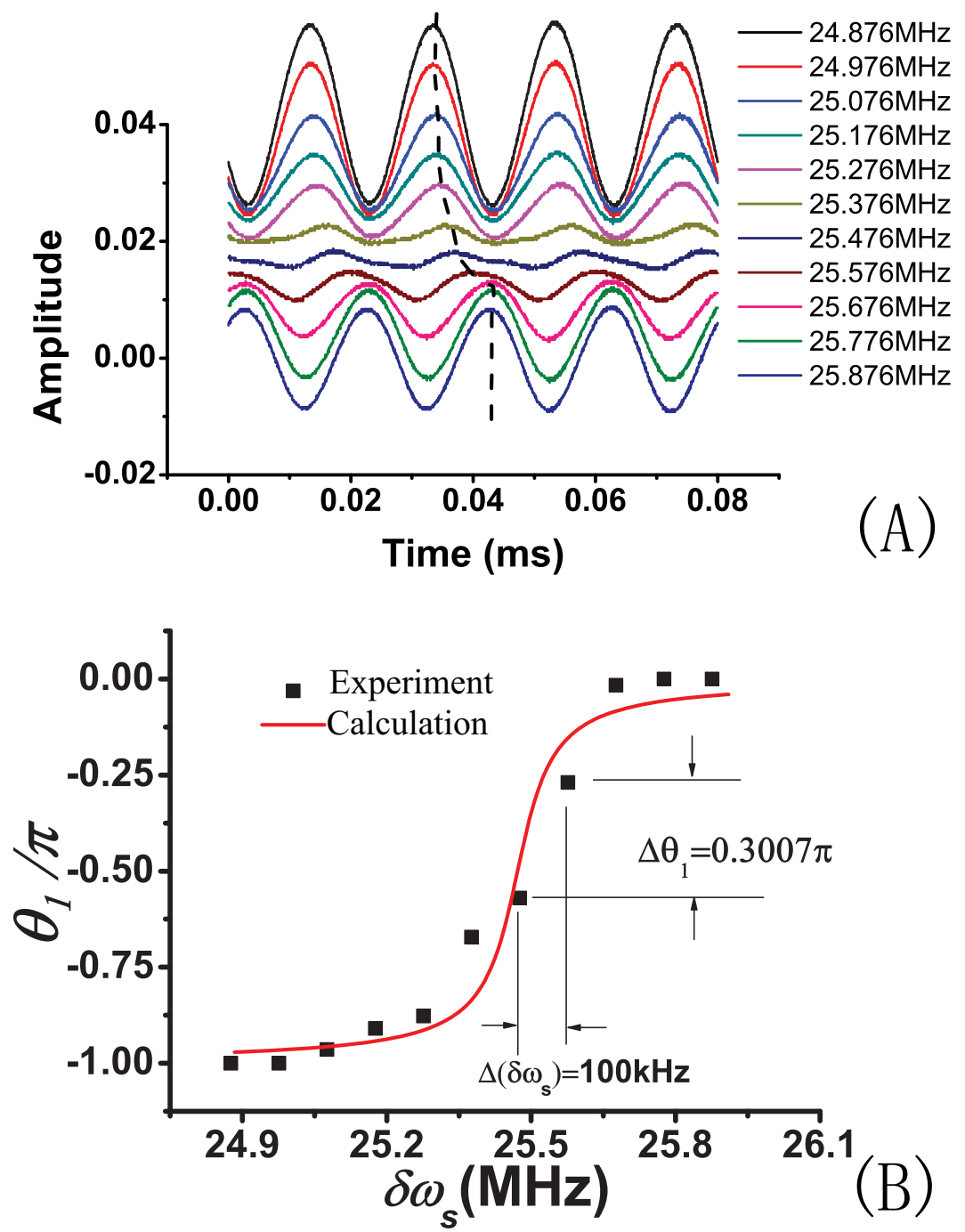

Fig. 5. The output first harmonic wave measured at different frequency detunings and the normalized phase shift of the first harmonic wave together with the simulation. The black dash curve in (A) shows the evolution of the modulation phase when the frequency of signal is detuning. The normalized phase shift is shown in (B). The red solid curve is the calculation with $\hat{G}_{0}=3.245$ and $\varphi_{2}=1.0009 \pi$ and the sensing window is around $450 \mathrm{kHz}$. 
the first harmonic, the amplitude of which approaches zero near the maximum sensitivity point. The frequency window of large sensitivity is $\sim 450 \mathrm{kHz}$, within which the normalized phase shift changed from 0.9 to 0.1 . By fitting the experimental result to the theoretical phase shift given by Eq. (7), which is presented as the red solid curve in Fig. 5(B), we deduce values of $\hat{G}_{0}=3.245$ and $\varphi_{2}=1.0009 \pi$, thereby confirming that the sensing window lies in the fast-light region.

\section{Conclusion}

In conclusion, we have demonstrated a high spectral sensitivity on-chip Brillouin MMXGM frequency sensor. A spectral sensitivity of $\sim 9.5 \mathrm{mrad} / \mathrm{kHz}$ was achieved for a $50 \mathrm{kHz}$ modulation in the fast-light regime, which is of comparable sensitivity to km-long fiber experiments. This demonstration paves the way for photonic integration of high sensitivity spectral sensors.

\section{Acknowledgments}

This work was funded by the Australian Research Council (ARC) through its Discovery grant (DP1096838), Future fellowship (FT110100853), Laureate Fellowship (FL120100029) and Center of Excellence CUDOS (CE110001018). All experiments were performed in the laboratory of Prof Eggleton at the University of Sydney. The visit of corresponding author to USYD is supported by the 973 Programs (2013CB328702, 2011CB922003, and 2010CB934101), the 111 Project (B07013), the Natural Science Foundation of Tianjin (12JCQNJC00900 and 12JCQNJC00800), and the International S\&T Cooperation Program of China (2011DFA52870). 